\title{
Il y a critique et critique : épistémologie des modèles d'argumentation
}

There is Critique and there is Critique: Epistemology of Argumentation Models

\section{Emmanuelle Danblon}

\section{(2) OpenEdition}

12 Journals

\section{Édition électronique}

URL : http://journals.openedition.org/aad/1395

DOI : $10.4000 /$ aad. 1395

ISSN : 1565-8961

Éditeur

Université de Tel-Aviv

Référence électronique

Emmanuelle Danblon, " II y a critique et critique : épistémologie des modèles d'argumentation », Argumentation et Analyse du Discours [En ligne], 9 | 2012, mis en ligne le 15 octobre 2012, consulté le 20 février 2021. URL : http://journals.openedition.org/aad/1395; DOI : https://doi.org/10.4000/aad.1395

Ce document a été généré automatiquement le 20 février 2021.

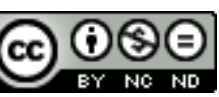

Argumentation \& analyse du discours est mis à disposition selon les termes de la licence Creative Commons Attribution - Pas d'Utilisation Commerciale - Pas de Modification 4.0 International. 


\title{
Il y a critique et critique : épistémologie des modèles d'argumentation
}

There is Critique and there is Critique: Epistemology of Argumentation Models

\author{
Emmanuelle Danblon
}

Je porte ainsi en moi, sculptée depuis l'enfance, une sorte de statue intérieure, qui donne une continuité à ma vie, qui est ma part la plus intime, le noyau le plus dur de mon caractère

François Jacob, La statue intérieure

\section{Introduction}

1 Les modèles actuels de rhétorique, d'argumentation ou d'analyse du discours entretiennent chacun un lien d'héritage avec une tradition plus ancienne. Ce lien, plus ou moins explicite en fonction des courants, va cependant toujours déterminer une certaine conception de la critique. À partir de cette conception de la critique, l'on pourra se prononcer sur la façon dont le chercheur envisagera son activité d'analyse du discours et des débats qui constituent son objet de recherche. C'est à une clarification de cette question que le présent article invite. Il plaide ensuite en faveur du modèle rhétorique antique, tout en cherchant à renforcer encore la dimension technique et pratique de la discipline, (encore) présente chez Aristote.

\section{Critique et raison}

2 Posons que tout modèle qui vise à l'analyse du discours à caractère argumentatif et/ou rhétorique, décrit un usage de la parole liée à l'action. De même, toute théorie de la rhétorique réfléchit sur les liens entre la validité au sens large ( la valeur») et l'efficacité persuasive («l'influence »). Or, au centre des tensions toujours d'actualité 
entre validité et persuasion, la notion de critique intervient tôt ou tard. Mais celle-ci appartient-elle au locuteur engagé dans le débat ou au théoricien qui analyse le débat? Et en quoi cette activité de critique consiste-t-elle au juste? Est-elle nécessairement liée à la démarche des analyses du discours?

3 Je voudrais montrer que cette question ne saurait se résoudre sans un examen préalable de l'épistémologie et, même, de la culture des divers modèles argumentatifs et/ou rhétoriques des analyse du discours. Je défendrai pour ma part le modèle rhétorique tel qu'il fut pensé à ses débuts, dans toute sa dimension de pratique (technè), d'obédience interdisciplinaire.

Commençons, pour éclairer la notion de critique, par un commentaire lexicologique.

Le Trésor de la Langue Française la définit comme une «Capacité de l'esprit à juger un être, une chose à sa juste valeur, après avoir discerné ses mérites et ses défauts, ses qualités et imperfections ${ }^{1} »$.

6 Comme dans toute définition, on peut chercher à expliciter la vision des choses, la topique, "l'idéologie", comme l'appellent les modèles critiques, bref, les idées préconçues qui sont à l'œuvre. Or cette définition recèle un vocabulaire éclairant pour une certaine conception de la raison. En particulier, doivent attirer notre attention les termes « capacité », « esprit », et «juger ». Tout d'abord, une « capacité » touche à la nature de l'homme, et à des aptitudes qui seraient au moins en partie spontanées. Ensuite, cette capacité relève du domaine intellectuel ou spirituel : c'est, précise la définition, l'«esprit» qui est concerné par cette aptitude. Enfin, le jugement que produit cette capacité semble relever de critères théoriques ou tout au moins, théorisables.

7 Il me semble que cette définition de la critique est directement inspirée d'une conception moderne voire moderniste de la raison, laquelle est profondément antirhétorique. À cette conception, on gagne à opposer une vision plus "grecque » de la raison. En effet, dans le modèle hérité de la rhétorique grecque, le jugement que constitue la critique doit être replacé dans le cadre d'une action, laquelle est le produit d'une délibération, dont l'élaboration pratique est le résultat de l'apprentissage d'une technique. En outre, cette action de critique n'est pas réalisée par un "pur » esprit, mais par un individu socialement déterminé qui fait entrer en dialogue un ensemble de considérations parmi lesquelles sensations et émotions occupent une place de choix. C'est du moins la conception de la rhétorique que je souhaiterais défendre, laquelle me paraît la plus fidèle au modèle décrit par Aristote.

\section{Rhétorique et raison}

8 Dans la rhétorique d'Aristote, d'une façon remarquable, la discipline est définie à la fois (ou plutôt tour à tour) comme une technique et comme une faculté. Lisons tout d'abord cette première mise au point sur la visée de la rhétorique : « Il est donc manifeste que la rhétorique n'appartient pas à un genre défini mais, tout comme la dialectique..., et qu'elle est utile, et aussi que sa fonction propre n'est pas de persuader, mais de voir les moyens de persuader que comporte chaque sujet [...] » (Rhétorique, I, 1, 1355b). Voyons à présent une définition plus complète : «Admettons donc que la rhétorique est la faculté de découvrir spéculativement ce qui, dans chaque cas, peut être propre à persuader. Aucun autre art n'a cette fonction » (I, 2, 1355b). On remarquera que, dans les deux cas, 
le verbe qui dénote l'action produite par la rhétorique est "découvrir spéculativement" ce qui est propre à persuader. Cette expression est choisie par Médéric Dufour pour traduire le terme grec théôrèsai, dont l'acception est à la fois plus large et plus variée, pouvant aller de voir, à considérer, en passant par contempler ou découvrir. Nous y reviendrons dans un moment.

Grâce à cette digression philologique qui me paraît essentielle à la compréhension de la culture rhétorique pensée par Aristote, je proposerai de rapprocher cette définition de la rhétorique d'une certaine conception de la critique que je défendrai. Dans cette conception, la critique doit être vue comme une activité à la fois plus pratique et plus intuitive que celle dont nous avons hérité de la tradition d'abord platonicienne, ensuite cartésienne. Il s'agit en somme de prendre au sérieux l'intelligence intuitive et son exercice, afin d'expliciter un impensé de la raison moderne. Or c'est sans doute en partie ce tabou qui me paraît fonder aujourd'hui les grandes divergences de conceptions dans nos modèles rhétoriques et argumentatifs des analyses du discours.

\section{La raison intuitive}

10 Je propose ainsi, pour retourner à une conception plus pratique et plus « intuitionniste» de la critique, d'aborder cette notion depuis la définition de la rhétorique qui met l'accent sur l'activité de l'orateur dont la technique consiste à "découvrir spéculativement" (théorèsai), ce qui est propre à persuader. Ce qui peut relever de la connaissance intuitive dans le vocabulaire aristotélicien, trouve un lien avec le verbe grec qui a donné le Français «critique » : crinein qui signifie avant tout «choisir» entre plusieurs options. C'est ce que fait apparaître l'article de Sophie Klimis, qui analyse de près la portée de la racine théôrein. Pour elle, il y a dans l'acception du verbe une notion d'observation dans laquelle se niche une forme de reconnaissance pré-discursive et pré-inférentielle (Klimis 2003). Alors même que son analyse ne regarde pas la Rhétorique, elle montre que le champ dans lequel intervient ce vocabulaire aristotélicien part des sensations individuelles pour atteindre à la délibération politique, en passant par la sensation commune d'une idée d'humanité. Il y a donc un lien intime, on est tenté de dire "génétique ", entre sensation, action, éthique et délibération. À travers ce lien naturellement présent dans la culture grecque mais distendu pour nous, on perçoit le projet aristotélicien d'articuler les différents aspects d'une raison humaine qui, toujours, perçoit l'individu ancré dans une collectivité et dans l'action citoyenne. Ce qui m'intéresse ici est bien sûr d'attirer l'attention sur cette conception des choses pour envisager une conception de la critique à partir de cet héritage. Notons en outre que l'exercice de l'intelligence intuitive, chez Aristote, permet à l'individu, citoyen, intégré dans une collectivité, de pratiquer l'action délibérée, celle qui lui permettra d'agir avec discernement, et de prendre des décisions « éclairées ", si l'on veut bien pardonner l'anachronisme de l'expression. Dans cette vision des choses, la critique est l'action du citoyen qui s'exerce sur la place publique, au cœur de la délibération.

\section{Il y a critique et critique}

11 Ainsi, l'on voit clairement apparaître deux conceptions de la critique. L'une, héritée des Grecs et qui met l'accent sur l'action délibérée. L'autre, héritée de la Modernité et qui 
insiste sur le jugement intellectuel. C'est à partir de ces deux conceptions différentes de la critique que je propose à présent de considérer les modèles contemporains pour les analyses du discours. Chacun semble en effet avoir gardé des traits de ces deux visions de la critique qui héritent de deux conceptions parfois incompatibles entre elles de la raison humaine.

Pour les uns, la critique est donc un jugement qui doit s'opérer à la suite d'une analyse menée par des experts, mais elle doit avoir une utilité directe dans la vie sociale. On reconnaît là un courant de tradition platonicienne, que l'on retrouve dans les modèles actuels qualifiés de "normatifs ». Pour les autres, la critique est l'action des citoyens dans la vie civile. Elle n'est donc pas nécessairement opérée par des experts. L'analyse théorique s'y trouve séparée du champ de l'action citoyenne et se donne généralement pour visée une "description» théorique des échanges argumentés. On retrouve l'héritage des modèles linguistiques d'analyse des arguments, dont la vocation n'est pas prescriptive et entend s'abstenir de tout jugement sur les échanges, pour ce qui est de leur dimension politique. Mais on sait que la frontière entre ces deux écoles est parfois poreuse, comme nous allons à présent le découvrir.

\section{La Critical Discourse Analysis}

Pour aborder à présent la conception défendue par les modèles anglo-saxons de Critical Discourse Analysis (CDA), je proposerai en guise d'incipit une mise en discussion du point de vue de Ruth Wodak (2009) lorsqu'elle expose l'épistémologie et l'héritage intellectuels de ces modèles. Le courant, rappelons-le, a été initié par Norman Fairclough en Angleterre, dans les années $80^{2}$. Les mises au point de Wodak me permettront en même temps de décrire la culture dont se réclame ce courant des analyse du discours, et de me donner une série de critères par rapport auxquels je pourrai situer la position descriptive. Il me restera enfin à tenter de résoudre le problème de la frontière entre l'analyse théorique et l'action citoyenne dans le jeu de la critique.

En première remarque, il faut signaler que Wodak insiste sur l'importance de la dimension interdisciplinaire pour l'analyse du discours. Elle cite: l'histoire, la sociologie, les sciences politique, une théorie des genres, la communication et enfin la linguistique (101-102). Je reviendrai, lors de l'exposé du modèle rhétorique, à la liste des disciplines qui devraient entrer dans le partenariat pour un projet des analyses du discours. L'auteur en vient ensuite à problématiser la notion de «critique » de la façon suivante :

La notion de "critique» porte des significations très différentes pour chacun: certains chercheurs adhèrent aux idées de l'Ecole de Frankfort, d'autres à un courant de la critique littéraire, d'autres encore à la pensée de Marx [...]. Fondamentalement, l'adjectif « critique » peut être compris comme le fait de garder une distance par rapport aux données, de les contextualiser socialement, de rendre les positions politiques respectives explicites et d'être capable d'un retour sur soi en tant qu'universitaire qui entreprend une recherche. Pour tous ceux qui s'intéressent à la $\mathrm{CDA}$, le caractère applicatif des résultats est important, que ce soit en direction des enseignants, des médecins ou des officiels dans l'organisation de séminaires pratiques, dans l'écriture d'opinions expertes ou encore dans l'élaboration de manuels scolaires ${ }^{3}$. 
On retient de cette définition de la critique qu'elle constitue bien la cheville ouvrière de l'analyse, laquelle est opérée par un/e universitaire qui, de par son expertise, est capable de "garder » une distance vis-à-vis des données. En outre, cette analyse doit être mise au service de la société civile en ce qu'elle doit servir de guide pour aider les acteurs sociaux dans leur pratique de la délibération. C'est d'ailleurs dans des termes très clairs que cette visée de l'analyse est ensuite expliquée :

Les théories critiques, dont fait partie la CDA, se voient offrir un statut particulier, celui de guides pour l'action humaine. Elles ont pour but d'éclairer par la connaissance et d'apporter leur soutien à une démarche d'émancipation. Ces théories ne cherchent pas seulement à décrire et à expliquer, mais également à déraciner une certaine forme d'aveuglement.

On ne peut dire plus clairement que le but de l'analyse des experts, grâce à la démarche critique, consiste à éclairer le citoyen dans ses points de vue sur les débats.

\section{Les réticences adressées aux modèles normatifs}

Dans un article de 2004, Marianne Doury (2004) a clairement énoncé les critiques que l'on peut adresser à tout modèle d'analyse qui prétend " éclairer " le débat public à partir d'une position d'expertise et des analyses que celle-ci autorise. De cette critique, il faut retenir à ce stade deux éléments essentiels. Le premier est celui de l'impossible neutralité du chercheur, surtout dans des matières à forte tonalité polémique. En outre, le choix - toujours tentant pour le chercheur - de ces débats à tonalité passionnée pose le problème des réactions émotionnelles que les données susciteront nécessairement chez le lecteur. Il en est d'ailleurs ainsi de Wodak qui choisit d'analyser un discours de Jörg Haider, chef de file du parti d'extrême droite autrichien, pour illustrer son modèle. On a peine à croire, comme le note Marianne Doury à propos de corpus similaires, que le lecteur ne se range pas spontanément à l'analyse d'un chercheur qui dénoncerait un discours anti-démocrate comme « infâmant ». L'on voit ainsi les limites d'une analyse qui prend le risque de distribuer d'emblée les bons points et les mauvais points, non pas à partir d'une critique basée sur une expertise scientifique mais sur une position politique difficilement discutable pour les démocrates que nous sommes tous supposés être.

Il reste qu'aux yeux des partisans d'une CDA, la question de la responsabilité du chercheur et de son engagement dans la vie civile reste entière. Enfin, comme je l'ai évoqué plus haut, la controverse entre partisans d'un modèle évaluatif et d'un modèle descriptif des analyses du discours est bien souvent plus fine et plus nuancée qu'on ne veut bien le reconnaître.

\section{Que faire de la critique?}

19 Je ne citerai que deux auteurs qui témoignent du caractère nuancé de ces positions, lequel nous oblige, je crois, à poursuivre le dialogue entre les différentes écoles d'analyse du discours quant à leur visée ultime, pour la recherche et pour l'action citoyenne. Le premier, Michael Leff ${ }^{4}$, dans le domaine américain, témoigne d'une importante recherche inspirée du modèle rhétorique classique, dans laquelle l'attention portée au détail du texte et à sa réalité historique est sans équivoque. Simultanément, Leff entendait mettre ses compétences rhétoriques au service de 
l'action et de la formation des citoyens, de façon à améliorer leur capacité à argumenter de façon «éclairée». Dans cette tradition, l'utilité publique des compétences théoriques en matière de rhétorique et d'argumentation ne prête pas à discussion. Il y aurait même à ses yeux une certaine frilosité chez le chercheur qui refuserait cet ancrage citoyen ${ }^{5}$.

Un autre auteur, à présent, venu de France, qui pourrait présenter des traits oxymoriques avec ceux des modèles d'Outre-Atlantique. Il s'agit de la linguiste MarieAnne Paveau dont les travaux consistent au départ à décrire et à analyser certains types d'usages linguistiques et discursifs. Comme tout linguiste qui se respecte, Paveau prend bien soin de ne pas confondre description et évaluation dans la démarche d'analyse des productions discursives dans lesquelles elle s'engage. Or, comme Michaël Leff, elle a comme une « double vie » d'intellectuelle et de citoyenne. Voici comment, et voici pourquoi, je propose de qualifier son exemple d'oxymorique par rapport à celui de Michael Leff. Contrairement à celui-ci, Marie-Anne Paveau n'entreprend pas de former la jeunesse française à un bon usage du débat public. En revanche, sur un blog ${ }^{6}$ qu'elle anime depuis quelques années, la parole circule selon certains critères d'éthique de la discussion qui font eux-mêmes l'objet de sa recherche, par ailleurs ${ }^{7}$. Ici donc, le lien entre théorie et pratique existe bien dans le chef du chercheur mais selon une modalité plus inhabituelle, plus singulière, que celle que l'on trouve souvent Outre-Atlantique, où la responsabilité du chercheur est spontanément engagée sur la place publique.

Ces deux exemples n'ont d'autre objectif que d'illustrer la grande variété et l'éventail des possibilités qui s'offrent au chercheur qui entreprend de mettre sa réflexion au service des discours en lien avec l'action publique. Ainsi, des positions les plus spontanément normatives à celles qui se concentrent en principe sur les usages réels, rares sont les chercheurs dans nos disciplines qui ne se sentent pas aussi concernés par la question des prises de position politique, sa légitimité du point de vue de l'analyste, mais aussi son opportunité du point de vue du citoyen.

Cette problématisation de la question me conduit, à présent, à tenter de clarifier ma position sur la question des analyses du discours.

\section{Qu'entendons-nous par « analyser »?}

Voyons tout d'abord, de ce point de vue, comment l'on peut comprendre l'activité d'analyse selon un modèle comme celui de la CDA. Dans une telle perspective, on dira que l'analyse consiste précisément dans cette démarche critique formée par un jugement théorique. Plus précisément, au vu de ce qu'explique Ruth Wodak, la critique doit permettre de passer d'une vision des choses idéologique et stéréotypée à une autre, «objective " et comme "épurée» de ces "fausses croyances». La critique consiste donc en un travail de mise à distance des préjugés, facilitée par l'expertise du chercheur qui a appris à opérer cette "distinction» (crinein). Rappelons, une fois encore, que le résultat de cette purification linguistique et cognitive doit être directement utile aux citoyens et utilisable comme tel dans la vie sociale.

Dans un modèle qui respecterait un descriptivisme strict, la critique devrait être entièrement absente de l'analyse, laquelle devrait utiliser tous les outils théoriques qui appartiennent au champ dans lequel le chercheur travaille (linguistique, sociologie, psychologie, etc.). L'idéal de cette analyse doit en principe respecter les canons de la 
science moderne où, en tout cas, la dimension quantitative et reproductible est pertinente. Dans cette conception de la science qui, pour évoquer l'adage bien connu, sait "séparer l'oratoire du laboratoire ", le chercheur laisse ses opinions, y compris celles qu'il ne manque pas d'éprouver lorsqu'il analyse les débats, dans la sphère privée (l'oratoire) pour enfiler une blouse blanche imaginaire (qui symbolise le laboratoire) supposée garantir un rituel d'objectivation des ses points de vues. On a vu que cette position est critiquée pour son caractère peu vraisemblable au plan psychologique.

Il faut en tout cas reconnaître que les deux options, normative comme descriptive, présupposent une conception rationaliste de la raison, laquelle est fondée surtout sur des critères théoriques - celui d'un jugement de l'esprit - et cela, que l'on associe la critique à l'analyse ou que l'on dissocie les deux activités.

\section{Critique et analyse dans un modèle rhétorique}

Revenons finalement au modèle rhétorique et à la critique qu'il nous conduit nécessairement à adresser à une conception trop réductrice de la raison. Pour répondre finalement à la question de l'analyse, il convient de revenir à présent à ce champ lexical aristotélicien qui mêle les jugements théoriques à la décision pratique, l'intelligence intuitive à l'exercice du raisonnement. L'ensemble de ces traits se trouve dans l'acception du terme théôrèsai qui définit l'activité de celui qui pratique la rhétorique. $\mathrm{Au}$ fond, la définition d'Aristote montre d'emblée qu'il y a deux activités, l'une plus pratique, l'autre plus théorique. La première concerne surtout le citoyen qui doit persuader et être persuadé en retour. La seconde concerne davantage le spécialiste, orateur lui aussi mais souvent enseignant et expert en la matière. Sa spécialité, son expertise, est de savoir comment " découvrir spéculativement " (théôrèsai) ce qui, dans chaque cas, sera propre à persuader. Ainsi, dans un tel modèle, les sphères publique et privée, scientifique et pratique, ne sont pas aussi cloisonnées qu'ailleurs. Certes, c'est surtout comme citoyen que l'orateur exercera la critique, qui, dans ce cas-là, ne sera pas une analyse théorique mais un savoir pratique formalisé dans une technique qui le guide dans sa recherche du bon choix (crinein). Ainsi, l'agilité de l'orateur sera d'autant plus développée qu'elle sera exercée au sein de la technique rhétorique elle-même. Mais l'on voit tout de suite que le modèle antique ne prévoit guère de place à une " analyse » purement spéculative dont les résultats seraient directement utiles à une théorie rhétorique. En somme, on doit admettre qu'Aristote se concentre davantage sur la production que sur l'analyse des échanges d'arguments. C'est donc aussi à cette lacune qu'un modèle contemporain de théorie rhétorique doit remédier.

Personnellement, pour réaliser une telle analyse, j’y mettrais les éléments suivants. Une phase de microlecture comme celle prônée par Michaël Leff. Une description patiente de la topique, par la recherche d'indices textuels tels que ceux de la polyphonie et de l'intertextualité. Une analyse pragmatico-rhétorique qui tienne compte surtout des genres, des preuves, des différents dispositifs fictionnels et des figures au sens très large. Voilà pour le fondamental.

Mais il convient ensuite d'engager cette analyse aussi serrée que rigoureuse dans une entreprise interdisciplinaire qui, en principe, ne sépare pas les sciences de l'homme des sciences de la nature. Aujourd'hui, une partie importante des sciences cognitives étend sa recherche à une conception moins réductionniste de la raison que celle héritée d'un certain cartésianisme. Le moment est ainsi venu de considérer des activités évidentes 
pour les Grecs mais à peine pensables pour nous, comme celles de théôrèsai ${ }^{8}$. Elles nous permettront, je crois, de comprendre d'une façon plus réaliste mais aussi plus apaisée ce que fait l'homme lorsqu'il fait de la rhétorique. Elles nous permettront peut-être aussi de renouer avec une certaine tradition humaniste dans laquelle on ne rechignait pas à être tour à tour philosophe, biologiste, anthropologue et... orateur.

\section{BIBLIOGRAPHIE}

Amossy, Ruth. (éd.). 2011. L’analyse rhétorique aux Etats Unis. Hommage à Michael Leff, Argumentation et Analyse du discours 6.

Amossy, Ruth \& Roselyne Koren. (éds.). 2004. Argumentation et prises de position : pratiques discursives, Semen 17

Aristote. 1967. Rhétorique, Traduction et commentaire établis par Médéric Dufour, 3 vol. (Paris : Les Belles Lettres)

Doury, Marianne. 2004. «La position du chercheur en argumentation », Amossy, Ruth \& Roselyne Koren (éds.), Argumentation et prises de position : pratiques discursives, Semen 17

Fairclough, Norman. 1989. Language and Power (Londres : Longman)

Klimis, Sophie. 2003. « Voir, regarder, contempler : le plaisir de s'apprendre son humanité », La poétique d'Aristote : les lectures morales et politiques de la tragédie, Les Etudes Philosophiques, 466-482

Paveau, Marie-Anne. 2011. «Les diseurs de vérité 6. Prendre/donner la parole. Dialogue avec Luc Boltanski », La pensée du discours, http://penseedudiscours.hypotheses.org/1191.

Petitmengin, Claire. 2001. L'expérience intuitive, préface de Francisco Varela. (Paris : L'Harmattan) Wodak, Ruth. 2009. "Pragmatique et Critical Discourse Analysis : un exemple d'une analyse à la croisée des disciplines », Semen, 27, «Critical Discourse Analysis I. Les notions de contexte et d'acteurs sociaux ", Schepens, Philippe \& Adèle Petitclerc (éds), pp. 97-125

\section{NOTES}

1. On peut consulter le Trésor de la Langue Française en ligne à l'adresse : http://atilf.atilf.fr

2. Je ne cite ici que l'ouvrage fondateur de Norman Fairclough, 1989, Language and Power (Londres: Longman)

3. Wodak, op. cit., p. 8. La citation suivante ibid.

4. On se reportera au volume édité par Ruth Amossy, 2011, «L'analyse rhétorique aux Etats Unis. Hommage à Michael Leff ", Argumentation et Analyse du discours 6.

5. Je me souviens ainsi d'une conversation avec Michael Leff, lors d'une rencontre organisée par Ruth Amossy. Cet homme très ouvert écoutait attentivement mes arguments en défense d'un modèle descriptiviste. Il semblait être en partie convaincu au plan théorique. Mais il m'a exprimé ensuite combien il trouvait dommage, triste, pour tout dire, consternant, que nous décidions 
ainsi de ne pas assumer de responsabilité vis-à-vis de la société sur la question des débats. Il m'est impossible d'oublier cette conversation.

6. On peut découvrir la richesse et le dynamisme des interventions à l'adresse: http:// penseedudiscours.

7. Pour aller jusqu'au bout de la coïncidence, on lira en particulier un échange entre Marie-Anne Paveau et le sociologue Francis Chateaureynaud à propos de l'ouvrage de Luc Boltanski : De la critique. Précis de sociologie de l'émancipation (Paris: Gallimard) 2009. À mes yeux, l'ironie est double. Tout d'abord, la controverse entre les deux chercheurs s'oriente assez rapidement sur des questions d'éthique de la discussion, l'ouvrage de Boltanski devenant finalement un prétexte à l'échange. Mais ensuite, cet ouvrage qui porte précisément sur la critique, ne permet pas, à mon sens, de nourrir notre propre débat sur la critique, trop préoccupé qu'il est à se situer par rapport à ses illustres prédécesseurs : Althusser, Foucault, Bourdieu.

8. Citons pour seul exemple l'excellent livre de Claire Petitmengin, L'expérience intuitive (2001). On $\mathrm{y}$ voit se développer une réflexion dont la profondeur et la sensibilité ne font en aucune façon obstacle à la scientificité.

\section{RÉSUMÉS}

Cet article part d'un constat. La conception de la critique est toujours liée à l'épistémologie (souvent implicite) des modèles d'analyse du discours. C'est à ce niveau que se situe le débat entre experts de ces disciplines. Je défends ensuite le modèle rhétorique en proposant d'insister sur sa dimension pratique laquelle est directement liée aux émotions et aux intuitions. Ainsi, la rhétorique est avant tout une technique est c'est dans cet aspect de la discipline qu'il faut chercher le cœur de sa rationalité. Je proposerai finalement d'ajouter ces nouveaux critères à une conception élargie de la raison humaine qui tienne compte également d'outils contemporains pour les analyses du discours.

This paper claims that any conception of critique is necessarily linked with the (often implicit) epistemology of models for Discourse Analysis. This is what actually underlies the debates of the experts in the discipline. I present here a defense of the rhetorical model - but at the same time, I propose to highlight its practical dimension, which is closely connected to emotions and intuitions. Thus rhetoric is, primarily, a technique. This aspect of the discipline lies at the very heart of its rationality. Such criteria should be added to an expanded conception of human reason which would take into account contemporary tools for Discourse Analysis.

\section{INDEX}

Mots-clés : critique, épistémologie, humanisme, intuition, persuasion, raison humaine, technique

Keywords : critique, epistemology, humanism, intuition, persuasion, human reason, technique 
AUTEUR

EMMANUELLE DANBLON

Université Libre de Bruxelles, GRAL 